\title{
Relationship between Total Cholesterol and High-Density Lipoprotein Cholesterol and the Effects of Physical Exercise, Alcohol Consumption, Cigarette Smoking and Body Mass Index
}

\author{
Hiroshi Koyama, ${ }^{1}$ Masayuki OgawA, ${ }^{2}$ and Shosuke SUzUKI ${ }^{2}$ \\ ${ }^{1}$ Department of Environmental Health Sciences, Tohoku University \\ School of Medicine, Seiryo-machi, Aoba-ku, Sendai 980, Japan \\ ${ }^{2}$ Department of Public Health, Gunma University School of Medicine, \\ Showa, Maebashi 371, Japan
}

(Received January 31, 1990)

\begin{abstract}
Summary Despite the fact that high-density lipoprotein cholesterol (HDLC) is a part of total cholesterol (TC), the serum level of this portion has been reported to have no or only a weak relationship to the TC level. The present study assessed the relationship between HDLC and TC considering alcohol consumption, cigarette smoking, and body mass index (BMI) in 366 male workers classified into three groups by the habitual physical exercise. The results showed the different effects of alcohol consumption, cigarette smoking, and BMI on the level of HDLC among these three groups, and alcohol consumption lowered the LDLC level only in the exercise group. The closest relationship between HDLC and TC was seen in the exercise group, even after taking other factors into account. The result suggests that the HDLC level must be evaluated relative to the level of TC. As an indicator of serum lipid patterns the validity of the ratio of HDLC to TC (HDLC/TC) was discussed.
\end{abstract}

Key Words alcohol consumption, body mass index, cigarette smoking, HDL-cholesterol, LDL-cholesterol, physical exercise, total cholesterol

The inverse relationship between total serum cholesterol (TC) and highdensity lipoprotein cholesterol (HDLC) in coronary heart disease (i.e., high TC and low HDLC relate to the development of the disease) has been well recognized $(1,2)$. Although HDLC is a part of serum cholesterol, the level of HDLC has been reported to have no or only a weak relationship with the level of TC (3-5).

The factors affecting these cholesterol levels are different. Genetic determinants have a greater influence on the level of TC than HDLC (6), and TC is further

1 小山 洋, ${ }^{2}$ 小川正行, 鈴木庄亮 
affected by dietary cholesterol intake (7-9) and amount and kinds of dietary fats (9-11), which do not affect the HDLC level $(9,11)$. On the other hand, the HDLC level positively correlates with physical exercise $(12,13)$ and alcohol consumption (14-16), and is inversely related to cigarette smoking $(15,16)$ and the amount of body fat (17).

Low-density lipoprotein cholesterol (LDLC) is the major part of serum cholesterol and the level of TC generally reflects this portion. The purpose of this paper is to examine the effects of physical exercise, body mass index (BMI), alcohol intake, and cigarette smoking on the cholesterol levels of LDL and HDL and to discuss the appropriate indicators to evaluate the effects of these factors on the lipid patterns.

\section{SUBJECTS AND METHODS}

Participants for the study were 366 males aged 40 to 61 years involved in sedentary or manual labor in an electric appliance factory in Japan. Measurement of body height and weight for calculation of BMI (weight for height ${ }^{2}$ ), and a questionnaire for habitual alcohol consumption, cigarette smoking, and physical exercise, were conducted in January, 1987.

Blood samples were taken in the morning before breakfast using vacuum tubes without any anticoagulants. The samples were then centrifuged to separate sera and stored at $4{ }^{\circ} \mathrm{C}$. All analyses were completed within one week. HDLC was measured using the heparin-manganese precipitation method (HDL-cholesterolTest, Wako). TC and triglycerides (TG) were assayed with enzymatic colorimetric methods (Cholesterol C-Test, Wako, and Triglyceride-Test, Wako). The level of LDLC was estimated from TC, HDLC and TG with the formula of Friedewald $(\mathrm{LDLC}=\mathrm{TC}-\mathrm{HDLC}-\mathrm{TG} / 5)$.

The participants were classified into three groups according to the frequency and intensity of their physical exercises. The kind of exercise and its duration (minutes) and frequency (days per week) were obtained from the questionnaire (18). Subjects not participating in habitual exercise were classified into the non-exercise group (225 men). The remaining subjects performed a total of 19 kinds of physical exercises. These exercises were divided into two groups according to the intensity expressed by the relative metabolic rate (RMR) (19); i.e., a moderate or more intensive exercise (e.g. jogging or swimming) group ( $R M R>5$ ), and a mild exercise (e.g. walking) group $(\mathrm{RMR}<5)$. Since the elevated levels of HDLC have been reported in subjects who performed moderate exercise for at least 30 min per day and 3 days per week (20-25), the subjects who met these criteria were classified into the exercise group ( $28 \mathrm{men}$ ). The other 113 men who were practicing mild and/or moderate exercise infrequently (less than 3 days per week) or of short duration (less than $30 \mathrm{~min}$ ) were classified into the intermediate exercise group.

In the questionnaire the kind of alcoholic beverages, the quantity per day, and 
the frequency per week were asked. The quantity was asked using common consumer's measures such as glasses or bottles. A bottle of beer, $180 \mathrm{ml}$ of Japanese saké, and two glasses of single whisky were regarded to contain the same quantity of alcohol and were each counted as 1 unit of consumption. Weekly intake of alcohol was calculated with this unit and the consumption was expressed by the average unit per day.

The analysis of variance was used for comparison of the means of the three groups, and $t$-test with the Ryan method was used for comparison of two means, if necessary. The chi-square test was used for comparison of the frequency distribution of alcohol drinking and cigarette smoking of the three groups. The multiple regression analysis was used to examine the relationship between the cholesterol levels and the affecting factors.

\section{RESULTS}

Frequency distribution of each group by alcohol consumption is shown in Table 1. In any group, one-forth or -fifth were non-drinkers and the most of the drinkers consumed less than 2 units of alcohol per day. The distribution pattern of each group did not differ from each other. Table 2 shows the frequency distribution of the number of cigarettes smoked by the three groups. The percentage of non-smokers was relatively higher in the exercise group than in the other two

Table 1. Frequency distributions of habitual alcohol intake of the non-exercise (NON), intermediate (INT), and exercise (EXE) groups.

\begin{tabular}{lrrr}
\hline Alcohol & \multicolumn{1}{c}{ NON } & \multicolumn{1}{c}{ INT } & \multicolumn{1}{c}{ EXE } \\
\hline 0 & $56(24.9 \%)$ & $29(25.7 \%)$ & $6(21.4 \%)$ \\
$<2$ & $109(48.4 \%)$ & $62(54.9 \%)$ & $17(60.7 \%)$ \\
$2-$ & $60(26.7 \%)$ & $22(19.5 \%)$ & $5(17.9 \%)$ \\
\hline Total & 225 & 113 & 28 \\
\hline
\end{tabular}

Alcohol (units/day): A bottle of beer, $180 \mathrm{ml}$ of Japanese saké, and two glasses of single whiskey are regarded to contain the same quantity of alcohol and are counted as 1 unit each. Chi-square value $=3.37(p=0.498)$.

Table 2. Frequency distributions of habitual cigarette smoking of the non-exercise (NON), intermediate (INT), and exercise (EXE) groups.

\begin{tabular}{lrrr}
\hline Cigarettes & NON & INT & \multicolumn{1}{c}{ EXE } \\
\hline 0 & $90(40.0 \%)$ & $56(49.6 \%)$ & $19(67.9 \%)$ \\
$1-19$ & $35(15.6 \%)$ & $16(14.2 \%)$ & $3(10.7 \%)$ \\
$20-$ & $100(44.4 \%)$ & $41(36.3 \%)$ & $6(21.4 \%)$ \\
\hline Total & 225 & 113 & 28 \\
\hline
\end{tabular}

Chi-square value $=9.34(p=0.06)$. 
Table 3. The means and standard deviations of age, body mass index (BMI), total cholesterol (TC), LDL-cholesterol (LDLC), HDL-cholesterol (HDLC), triglycerides (TG), and the proportion of LDL-cholesterol to total cholesterol (LDLC/TC) and of HDL-cholesterol to total cholesterol (HDLC/TC) in the non-exercise (NON), intermediate (INT), and exercise (EXE) groups.

\begin{tabular}{lcccl}
\hline Variable & NON $(n=225)$ & INT $(n=113)$ & EXE $(n=28)$ & $F$-value \\
\hline Age & $46.2 \pm 4.9$ & $45.1 \pm 4.7$ & $45.6 \pm 4.3$ & 1.96 \\
BMI & $22.5 \pm 2.5$ & $23.2 \pm 2.9$ & $21.7 \pm 1.9$ & $4.72^{\# \#}$ \\
TC & $203.6 \pm 39.2$ & $193.2 \pm 33.0$ & $186.5 \pm 22.7$ & $4.90^{\# \#}$ \\
LDLC & $126.6 \pm 36.6$ & $117.4 \pm 27.8$ & $111.0 \pm 16.8$ & $4.77^{\# \#}$ \\
HDLC & $49.5 \pm 13.3$ & $50.1 \pm 16.2$ & $57.8 \pm 14.4^{*}$ & $4.17^{\#}$ \\
TG & $137.6 \pm 79.2$ & $128.4 \pm 63.2$ & $88.6 \pm 35.5^{*}$ & $5.87^{\# \#}$ \\
LDLC/TC & $61.3 \pm 8.9$ & $60.4 \pm 7.2$ & $59.5 \pm 4.9$ & 0.86 \\
HDLC/TC & $25.3 \pm 8.5$ & $26.3 \pm 8.3$ & $30.9 \pm 6.5^{*}$ & $5.81^{\# \#}$ \\
\hline
\end{tabular}

* The difference is significant at $p<0.05$ in comparison with the non-exercise and intermediate groups ( $t$-test with the Ryan method). ${ }^{\#} F$-value is significant at $p<0.05$ (analysis of variance). \#\# significant at $p<0.01$.

Table 4. The correlation coefficients between total cholesterol (TC), HDL-cholesterol (HDLC), LDL-cholesterol (LDLC), and triglycerides (TG) in the non-exercise (NON), intermediate (INT), and exercise (EXE) groups.

\begin{tabular}{lccc}
\hline \multicolumn{1}{c}{ Relation } & NON $(n=225)$ & INT $(n=113)$ & EXE $(n=28)$ \\
\hline LDLC-TC & $0.914^{* * *}$ & $0.875^{* * *}$ & $0.843^{* * *}$ \\
LDLC-TG & $0.156^{*}$ & $0.185^{*}$ & 0.086 \\
HDLC-TC & -0.055 & $0.271^{* *}$ & $0.563^{* *}$ \\
HDLC-TG & $-0.414^{* *}$ & $-0.404^{* *}$ & $-0.510^{* *}$ \\
LDLC-HDLC & $-0.244^{* *}$ & -0.077 & 0.117 \\
\hline
\end{tabular}

*, $p<0.05 ; * *, p<0.01 ; * * *, p<0.001$.

groups; however, the difference of the distribution patterns was not statistically significant.

The mean values and standard deviations of age, BMI, serum TC, HDLC, LDLC, and TG are shown in Table 3. Mean age did not differ among the three groups. The levels of BMI, TC, and LDLC of the exercise group were relatively low, but not statistically significant. The exercise group was significantly higher in HDLC and lower in TG than the other two groups ( $t$-test with the Ryan method). The proportion of HDLC to TC (HDLC/TC) was significantly higher in the exercise group than in the other two groups. However, the proportion of LDLC to TC (LDLC/TC) showed almost the same value in each group. The $F$-value of HDLC/TC among the three groups was greater than that of HDLC itself. The intermediate group indicated similar lipid values to the non-exercise group.

As Table 4 shows, LDLC strongly correlated with TC in all groups. The relationship between LDLC and TG was weak and also no significance was 
Table 5. Standardized regression coefficients (beta) in the multiple regression analysis of HDL-cholesterol (HDLC) with total cholesterol (TC), alcohol consumption, cigarette smoking, and body mass index (BMI) in the non-exercise (NON), intermediate (INT), and exercise (EXE) groups.

\begin{tabular}{lccc}
\hline Variable & NON $(n=225)$ & INT $(n=113)$ & EXE $(n=28)$ \\
\hline TC & 0.018 & $0.323^{* *}$ & $0.604^{* *}$ \\
Alcohol & $0.242^{* *}$ & $0.199^{*}$ & $0.410^{*}$ \\
Smoking & $-0.106^{*}$ & $-0.295^{*}$ & 0.023 \\
BMI & $-0.351^{* *}$ & $-0.393^{* *}$ & -0.053 \\
\hline$R$ & 0.408 & 0.531 & 0.704 \\
\hline
\end{tabular}

$R$ : Multiple correlation coefficient. ${ }^{*}, p<0.05 ;{ }^{* *}, p<0.01$.

obtained in the exercise group. The coefficient value between HDLC and TG of each group showed similar result, in which HDLC correlates inversely with TG. However, the relationship between HDLC and TC differed from each other. HDLC did not correlate with TC in the non-exercise group, weakly correlated with TC in the intermediate group, and substantially correlated with $\mathrm{TC}$ in the exercise group. The inverse relationship between LDLC and HDLC was obtained only in the non-exercise group and no significant relation was shown in the other two groups.

The result of multiple regression analysis of HDLC with TC, alcohol consumption, cigarette smoking, and BMI is shown in Table 5. In the exercise group it was indicated that the level of TC was substantially important for determining the level of HDLC, and also in the intermediate group HDLC related slightly with the level of TC. However, in the non-exercise group the level of HDLC was not determined by the level of TC even after the effects of alcohol consumption, cigarette smoking, and BMI were taken into consideration.

The standardized correlation coefficients of alcohol consumption were positive and significant in all groups and the highest coefficient was obtained in the exercise group. Both cigarette smoking and BMI inversely affected the level of HDLC in the non-exercise and intermediate groups; however, this was not the case in the exercise group.

Table 6 shows the result of multiple regression analysis of LDLC with TC, alcohol consumption, cigarette smoking, and BMI in the three groups. The result showed that the level of LDLC was practically determined by only the level of TC in all groups. The other factors did not affect the level of LDLC except for alcohol consumption in the exercise group, where the level of LDLC decreased with the habitual intake of alcohol.

The distribution pattern of HDLC to TC was compared between the nonexercise group (dots) and the exercise group (circles) in Fig. 1. The non-exercise group was scattered diffusely, reflecting no relationship between HDLC and TC, while the exercise group showed a positive relationship. 
Table 6. Standardized regression coefficients (beta) in the multiple regression analysis of LDL-cholesterol (LDLC) with total cholesterol (TC), alcohol consumption, cigarette smoking, and body mass index (BMI) in the non-exercise (NON), intermediare (INT), and exercise (EXE) groups.

\begin{tabular}{lccc}
\hline Variable & NON $(n=225)$ & INT $(n=113)$ & EXE $(n=28)$ \\
\hline TC & $0.914^{* * *}$ & $0.865^{* * *}$ & $0.823^{* * *}$ \\
Alcohol & -0.112 & -0.041 & $-0.323^{* *}$ \\
Smoking & 0.028 & 0.037 & 0.090 \\
BMI & 0.020 & 0.086 & 0.012 \\
\hline$R$ & 0.920 & 0.880 & 0.904 \\
\hline
\end{tabular}

$R$ : Multiple correlation coefficient. ${ }^{* *}, p<0.01{ }^{* * *}, p<0.001$.

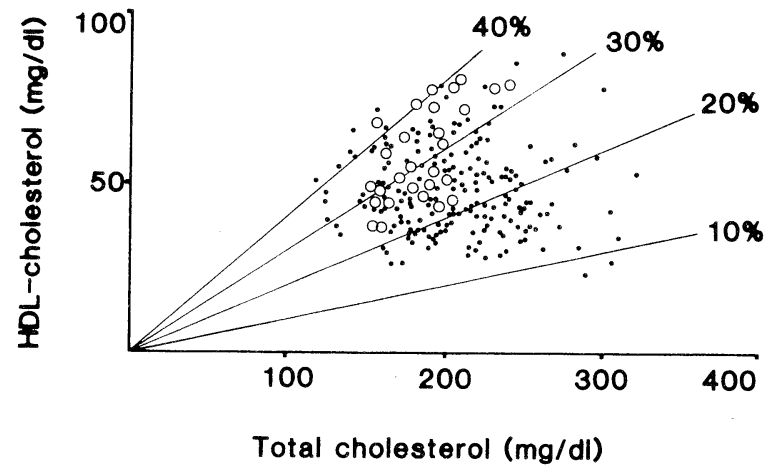

Fig. 1. Scattergram of total cholesterol and HDL-cholesterol in non-exercise (dots) and exercise (circles) groups, and the proportion lines of HDL-cholesterol to total cholesterol.

The lines drawn in the figure indicate the respective ratio of HDLC to TC. Most of the individuals of both groups were below the $40 \%$ line. The figure seems to indicate that the $40 \%$ is the limit of the proportion of HDLC to the respective level of TC. The proportion of HDLC of the exercise group rose near to the limit line, while the non-exercise group was distributed widely between $10 \%$ and $40 \%$ lines.

\section{DISCUSSION}

The habitual intake of alcohol, cigarette smoking, and BMI have been known as determinants of the HDLC level (14-17). The present results showed the different effects of these factors on the level of HDLC among the three groups by the habitual physical exercise. Cigarette smoking and BMI did not affect the HDLC level in the exercise group. Alcohol consumption raised the HDLC level more effectively in the exercise group than in the non-exercise and intermediate 
groups, and also lowered the LDLC level in the exercise group. Furthermore, the three groups differed in the relationship between HDLC and TC.

Although an inter-population study (26) has demonstrated a positive relationship between population averages of HDLC and TC, several lipid studies conducted within a single population have observed no or only a weak relationship between HDLC and TC (3-5). Sato et al. (27) have reported the positive correlations ( $r=0.317$ and 0.222 ) only in low TC subgroups (less than $180 \mathrm{mg} / \mathrm{dl}$ ). In the present study the exercise group showed closer relationship between HDLC and TC $(r=0.563)$, and the TC level of the group was not necessarily limited in low level, but ranged from 156 to $241 \mathrm{mg} / \mathrm{dl}$.

As low-density lipoprotein accounts for a greater portion of serum cholesterol and HDLC forms a part of TC, there must be a limit in the proportion of HDLC to TC. When HDLC increases and reaches the upper limit of the proportion, the HDLC level will be determined exclusively by the level of TC. As shown in Fig. 1, both non-exercise and exercise groups hardly exceed the $40 \%$ ratio line, which appears to be an upper limit of the proportion of HDLC to TC. In the exercise group, HDLC increased and nearly reached the upper limit, by which the positive relationship between TC and HDLC would be explained.

Figure 1 suggests that the level of HDLC must be evaluated in reference to the level of TC. The HDLC to TC ratio is considered useful as a referring method for the TC level. The ratio of HDLC/TC has already been used operationally to evaluate the effect of exercise on lipid patterns $(22,23)$ and also as an inverse predictor of the development of coronary heart disease $(2,28-30)$. The present study supports the significance of the HDLC/TC ratio for evaluating the effects of various factors on the lipid patterns.

The present study was financially supported by a grant from Uehara Life Science Foundation.

We thank Tai-ichiro Takemoto and Kazuhiko Moji, for their useful advice.

\section{REFERENCES}

1) Rhoads, G. G., Gulbrandsen, C. L., and Kagan, A. (1976): Serum lipoproteins and coronary heart disease in a population study of Hawaii Japanese men. N. Engl. J. Med., 294, 293-298.

2) Castelli, W. P., Garrison, R. J., Wilson, P. W. F., Abbott, R. D., Kalousdian, S., and Kannel, W. B. (1986): Incidence of coronary heart disease and lipoprotein cholesterol levels: The Framingham Study. J.A.M.A., 256, 2835-2838.

3) Castelli, W. P., Cooper, G. R., Doyle, J. T., Garcia-Palmieri, M., Gordon, T., Hames, C., Hulley, S. B., Kagan, A., Kuchmak, M., McGee, D., and Vicic, W. J. (1977): Distribution of triglyceride and total, LDL and HDL cholesterol in several populations: A cooperative lipoprotein phenotyping study. J. Chron. Dis., 30, 147-169.

4) Kesteloot, H., Lee, C. S., Park, H. M., Kegels, C., Geboers, J., Claes, J. H., and Joossens, J. V. (1982): A comparative study of serum lipids between Belgium and 
Korea. Circulation, 65, 795-799.

5) Haffner, S. M., Ava Knapp, J., Stern, M. P., Hazuda, H. P., Rosenthal, M., and Franco, L. J. (1985): Coffee consumption, diet, and lipids. Am. J. Epidemiol., 122, $1-12$.

6) Dahlen, G., Ericson, C., de Faire, U., Iselius, L., and Lundman, T. (1983): Genetic and environmental determinants of cholesterol and HDL-cholesterol concentrations in blood. Int. J. Epidemiol., 12, 32-35.

7) Keys, A., Anderson, J. T., and Grande, F. (1965): Serum cholesterol response to changes in the diet: II. The effect of cholesterol in the diet. Metabolism, 14, 759-765.

8) Connor, W. B., Cerqueira, M. T., Connor, R. W., Wallace, R. B., Malinow, M. R., and Casdorph, H. R. (1978): The plasma lipids, lipoproteins, and diet of the Tarahumara Indians of Mexico. Am. J. Clin. Nutr., 31, 1131-1142.

9) Chenoweth, W., Ullmann, M., Simpson, R., Leveille, G.(1981): Influence of dietary cholesterol and fat on serum lipids in men. J. Nutr., 111, 2069-2080.

10) Keys, A., Anderson, J. T., and Grande, F. (1965): Serum cholesterol response to changes in the diet: I. Iodine value of dietary fat versus 2S-P. Metabolism, 14, 747-758.

11) Becker, N., Illingworth, D. R., Alaupovic, P., Conner, W. E., and Sundberg, E. E. (1983): Effects of saturated, monounsaturated, and w-6 polyunsaturated fatty acids on plasma lipids, lipoproteins, and apoproteins in humans. Am. J. Clin. Nutr., 37, 355360.

12) Adner, M. M., and Castelli, W. P. (1980): Elevated high-density lipoprotein levels in marathon runners. J.A.M.A., 243, 534-536.

13) Moffatt, R. J., and Gilliam, T. B. (1979): Serum lipids and lipoproteins as affected by exercise: A review. Artery, 6, 1-19.

14) Brenn, T. (1986): The Tromso Heart Study: alcoholic beverages and coronary risk factors. J. Epidemiol. Community. Health., 40, 249-456.

15) Hully, S. B., Cohen, R., and Widdowson, G. (1977): Plasma high-density lipoprotein cholesterol level: Influence of risk factor intervention. J.A.M.A., 238, 2269-2271.

16) Donahue, R. P., Orchard, T. J., Kuller, L. H., and Drash, A. L. (1985): Lipids and lipoproteins in a young adult population. Am. J. Epidemiol., 122, 458-467.

17) Avogaro, P., Cazzolato, G., Bittolo Bon, G., Quinci, G. B., and Chinello, M. (1978): HDL-cholesterol, apolipoproteins A1 and B: Age and index body weight. Atherosclerosis, 31, 85-91.

18) Koyama, H., Ogawa, M., and Suzuki, S. (1989): Undou to Kessei HDL-cholesterol Ti tono Kanren. Jpn. J. Public. Health (in Japanese), 36, 33-37.

19) Koseisho (1984): Nihon Jin no Eiyo Shoyo Ryo. Daiichi Shuppan, Tokyo (in Japanese).

20) Huttunen, J. K., Lansimies, E., Voutilainen, E., et al.(1979): Effect of moderate physical exercise on serum lipoproteins: A controlled clinical trial with special reference to serum high-density lipoproteins. Circulation, 60, 1220-1229.

21) Brownell, K. D., Bachorik, P. S., and Ayerle, R. S. (1982): Changes in plasma lipid and lipoprotein levels in men and women after a program of moderate exercise. Circulation, 65, 477-484.

22) Liebman, M., Smith, M. C., Iverson, J., Thye, F. W., Hinkle, D. E., Herbert, W. G., Ritchey, S. J., and Driskell, J. A. (1983): Effects of coarse wheat bran fiber and exercise on plasma lipids and lipoproteins in moderately overweight men. Am. J. Clin. 
Nutr., 37, 71-81.

23) Baker, T. T., Allen, D., Lei, K. Y., and Willcox, K. K. (1986): Alterations in lipid and protein profiles of plasma lipoproteins in middle-aged men consequent to an aerobic exercise program. Metabolism, 35, 1037-1043.

24) Lampman, R. M., Santinga, J. T., Savage, P. J., Bassett, D. R., Hydrick, C. R., Flora, J. D., and Block, W. D. (1985): Effect of exercise training on glucose tolerance, in vivo insulin sensitivity, lipid and lipoprotein concentrations in middle-aged men with mild hypertriglyceridemia. Metabolism, 34, 205-211.

25) Fripp, R. R., and Hodgson, J. L. (1987): Effect of resistive training on plasma lipid and lipoprotein levels in male adolescents. J. Pediatr., 111, 926-931.

26) Knuiman, J. T., Hermus, R. J. J., and Hautvast, J. G. A. J. (1980): Serum total and high density lipoprotein (HDL) cholesterol concentrations in rural and urban boys from 16 countries. Atherosclerosis, 36, 529-537.

27) Sato, N., Miyazima, E., Suzuki, N., Ishikawa, T., Nakamura, H., Nagano, M., Ishii, K., Kagawa, Y., Hirano, T., Saga, M., and Iwasa, M. (1981): Lipoprotein cholesterol level in school-girls aged 16-18 with references to their nutrient intakes. J. Jpn. Soc. Food Nutr. (in Japanese), 34, 247-251.

28) De Backer, G., Rosseneu, M., and Deslypere, J. P. (1982): Discriminative value of lipids and apoproteins in coronary heart disease. Atherosclerosis, 42, 197-203.

29) Arntzenius, A. C., Kromhout, D., Barth, J. D., Reiber, J. H. C., Bruschke, A. V. G., Buis, B., van Gent, C. M., Kempen-Voogd, N., Strikwerda, S., and van der Velde, E. A. (1985): Diet, lipoproteins, and the progression of coronary atherosclerosis: The Leiden Intervention Trial. N. Engl. J. Med., 312, 805-811.

30) Castelli, W. P., and Anderson, K. (1986): A population at risk: Prevalence of high cholesterol levels in hypertensive patients in the Framingham Study. Am. J. Med., 80 (Suppl 2A), 23-32. 